\title{
Initial Hypothesis
}

\section{Editor's Note}

Since the 2nd half of the 16th century, over more than 300 hundred years of exploration and accumulation, research has entered the stage of revealing the nature of objects from observing the surface phenomena. Meanwhile, hypothesis-driven research (HDR) has gradually become the main form of research in many fields of modern science.

HDR begins with familiarizing the background knowledge, according to the direction of the problem, using rational thinking methods to generalize the known relevant scientific phenomena and laws and build scientific hypotheses, then carrying out theoretical deduction and prediction, and then designing corresponding research programs including scientific observation, scientific experiment or scientific investigation, so as to test hypotheses and further obtain the answers.

Usually, the formation of scientific hypothesis includes two stages: the initial hypothesis and the complete hypothesis. The former is only based on a small number of factual materials and existing relevant theories, and puts forward a tentative hypothesis for the answer of questions through thinking processing, which has not yet constituted a systematic discussion. Further, the initial hypothesis mainly includes the following four basic links: accumulating facts, mastering knowledge; analyzing facts, sorting out data; guessing, drawing conclusions; constructing concepts, expressing hypotheses. After putting forward the initial hypothesis, researchers should take this as the center, use various existing scientific theories and as many relevant background knowledge or conditions as possible to carry out extensive argumentation, explain the known facts, predict the unknown facts and potential rules or laws, and strive to theorize and systematize them, so as to expand the initial hypothesis into a relatively complete and stable theoretical system.

In brief, the formation of scientific hypothesis is a process of describing facts, explaining facts, and finding causal links and objective laws. Therefore, it requires objective analysis of problems, comprehensive collection of materials and in-depth processing, and on this basis, a comprehensive grasp of the links between things.

Considering the fact that the ability to propose hypotheses is an important feature of innovative talents, budding researchers should be trained from endowing them with capabilities for putting forward hypothesis. Just in this sense, CCR plans to select some hypothesis papers for publication to encourage more graduates or even undergraduates to express their special observation perspectives and innovative thinking on carcinogenesis, cancer prevention and treatment, further help them develop into mature researchers as soon as possible. The subsequent hypothesis is a good case in point.

\section{Effects of Pinus massoniana bark extract on the size of HeLa cells via Nesprin-2 pathway}

\author{
Xiaolu Zhang ${ }^{1,2^{\dagger}} \quad$ Mengqi $\mathrm{Li}^{1^{\dagger}} \quad{\text { Yingya } \mathrm{Li}^{1,3} \quad \text { Jiao Feng }}^{1,4} \quad$ Yingyu Cui ${ }^{1,5,6^{*}}$
}

\begin{abstract}
Proanthocyanidins (PAs) is the main constituent of Pinus massoniana bark extract (PMBE). PMBE was reported to induce cell cycle arrest and apoptosis in HeLa cells. During cell division, cells synthesize protein in G1 and G2 phases and replicate chromatin in S phase during interphase, which increases cell mass. Nesprins, a kind of protein encoded by syne gene, is a vital part of cytoskeleton and plays a role in cell cycle progress and cell division. HeLa cells were used as a model to examine effects of PMBE on cell growth and Nesprins expression with MTT assay and RT- PCR analysis, respectively. The cell size was evaluated by counting the cell number in a fixed area under microscope. The results showed that the size of survival HeLa cells in PMBE-treated group was obviously larger than that of those in control group $(\mathrm{p}=0.00223,<0.01)$, while the mRNA expression level of Nesprin-2 decreased significantly in PMBE-treated group $(\mathrm{p}=0.0201,<0.05)$. On this account, we put forward a hypothesis that PMBE inhibits the expression of syne-2, which leads to the decrease of Nesprin-2 and further results in the size increase of HeLa cells.
\end{abstract}

Keywords: Proanthocyanidins (PAs), Pinus massoniana bark extract (PMBE), cell size, HeLa cells, Nesprins

\footnotetext{
Received: March 20, 2020; Revised: June 12, 2020; Accepted: June 20, 2020; Published: June 25, 2020

* Correspondence to: Yingyu Cui, Key Laboratory of Arrhythmias of the Ministry of Education of China, Tongji University School of Medicine, Shanghai 200092, China; Email: yycui@tongji.edu.cn
}

\footnotetext{
$\dagger$ These authors contributed equally to this work and share first authorship

${ }^{1}$ Department of Regenerative Medicine, Tongji University School of Medicine, Shanghai 200092, China

${ }^{2}$ Department of gynecology and obstetrics, Jinshan Hospital of Fudan University, Shanghai 201508, China
} 


\section{Introduction}

Pinus massoniana Lamb, a member of Pinaceae family, is mainly grown in the south region of Changjiang River of China ${ }^{[1]}$. In traditional Chinese medicine, its bark, needles, pollen and turpentine are usually used to treat diseases including rheumatism arthralgia, hypertension, chilblain, and inflammation ${ }^{[1,2]}$. In addition, Pinus massoniana Lamb is rich in proanthocyanidins (PAs), which is a kind of natural antioxidant flavonoid ${ }^{[3,4]}$. In 2005, the components of Pinus massoniana bark extract (PMBE) were firstly reported as a natural extract developed and researched independently in $\mathrm{China}^{[2]}$. PMBE is a polyphenolic compound, whose primary active compound is PAs, including monomeric, oligomeric, and polymeric flavonoids. The B-type PAs, especially proanthocyanidin $\mathrm{B}_{3}$ is the main component of $\mathrm{PMBE}^{[5,6]}$.

PMBE was demonstrated to exhibit various bioactivities such as antiviral, anti-allergic, antioxidant and anticancer ${ }^{[2,7,8]}$. It has been proven that PMBE mainly induces cancer cells cycle arrest at $\mathrm{S}$ and G2/M phase ${ }^{[9]}$, further induces apoptosis of cancer cells via mitochondrial pathway and / or death receptor pathway ${ }^{[9-11]}$. While anthocyanins, metabolites of PAs heated in acidic media, can induce autophagy of human hepatocellular carcinoma (HCC) cells ${ }^{[11]}$. In addition, PMBE can also inhibit the migration and invasion of HeLa cell in vitro ${ }^{[12,13]}$. Obviously, the previous research of PMBE was focused on the inhibition of tumor cell growth and malignant behavior. The effect of PMBE on tumor cells' size has never been reported.

Nesprin was found in vascular smooth muscle cell in 2001, and there are 4 members of Nesprins encoded by syne-1 4 genes respectively in mammal. Nesprins are located in the nuclear envelope (NE), and their basic structures include C-terminal, ANC-1 and Syne/Nesprin homology (KASH), N-terminal-actin binding domain $(\mathrm{ABD})$ and spectrin repeat $(\mathrm{SR})^{[14]}$. The C-terminal of KASH protein interacts with inner nuclear membrane SUN-domain family members and the N-ABD can connect with F-actin. All of these components take part in constituting LINC complex ${ }^{[15,16]}$. The LINC spans both

\footnotetext{
${ }^{3}$ Center of Reproductive Medicine, Shanghai First Maternity and Infant Hospital, Tongji University School of Medicine, Shanghai 201204, China

${ }^{4}$ Department of Gastroenterology, Shanghai Tenth Peoples Hospital, Tongji University School of Medicine, Shanghai 200072, China

${ }^{5}$ Key Laboratory of Arrhythmias of the Ministry of Education of China (Tongji University), Shanghai 200120, China

${ }^{6}$ Institute of Medical Genetics, Tongji University School of Medicine, Shanghai 200092, China

Citation: Zhang XL, Li MQ, Li YY, et al. Effects of Pinus massoniana bark extract on the size of HeLa cells via Nesprin-2 pathway. Curr Cancer Rep, 2020, 2(1): 41-47.

Copyright: (c) 2020 Yingyu Cui, et al. This is an open access article distributed under the terms of the Creative Commons Attribution License, which permits unrestricted use, distribution, and reproduction in any medium, provided the original author and source are credited.
}

nuclear membranes, forming the bridge between nuclear lamina and cytoskeleton. Any depletion of the components of LINC complex will lead to cell mechanical tension decrease ${ }^{[17]}$. Nesprin-2 is located in both inner and outer nuclear membranes ${ }^{[14]}$. It has higher isogeny with Nesprin-1, and works in a similarly way. Nesprins located in outer nuclear membrane can help the nuclear and cytoskeleton position, promote the material transportation between nuclear and cytoplasm, power conduction and adjust the cytoplasmic network. Nesprin-2 links the nucleus to actin filaments by interacting with SUN1 \& $2^{[17,18]}$, connects with centrosome via microtubule and plays a role in cell polarization, and helps centrosome dislodge from nuclear membrane in the prophase of mitosis ${ }^{[19]}$.

Normally, cells have species specificity with a uniform size, which is regulated by nucleo-cytoplasmic ratio, ploidy, nutrition and so on ${ }^{[20]}$. Variability in cell size is affected by cell growth rate, cell-cycle length and asymmetry in cell division ${ }^{[21]}$. Increasing evidence indicates that cells can measure and regulate their size autonomously ${ }^{[21]}$. When the size of cell increases, its nucleo-cytoplasmic (N/C) ratio will decrease and gradually achieve the size threshold ${ }^{[21]}$. The cell enters $\mathrm{S}$ phase and starts to divide as soon as the $\mathrm{N} / \mathrm{C}$ ratio reduces to a specific threshold value. If the growth is blocked, cell cannot divide any more. However, this is not always the case for tumor cells $^{[20,22]}$, namely, when tumor cell division is blocked, tumor cell can grow as usually, thus huge cell and/or polyploid will appear, so-called giant tumor cells (GTC). Owing to mitotic disorders, GTC are possible to show inheritable drug resistance compared with typical small tumor cells ${ }^{[23-25]}$. Furthermore, for some kinds of tumor cells, tetraploid cells can revert to diploid, and acquire drug resistance and stronger viability ${ }^{[26]}$. Thus, the drugresistant giant cells could be potential reason for recurrence of tumor ${ }^{[27]}$. Therefore, the present study aims to explore the effects of PMBE on cell size in HeLa cells and potential mechanism, mainly focusing on the regulation of Nesprin-2.

\section{Results}

\subsection{Hypothesis}

Based on the results of our primary experiments: (1) the size of the cells in PMBE group was obviously larger than that of cells in blank group (Figure 1); (2) the transcription of gene syne-2 was significantly inhibited (Figure 2), we put forward a hypothesis that PMBE can regulate HeLa cell size through inhibiting the transcription of syne-2 gene (Figure 3). This may lead to the reduction of Nesprin-2 on nuclear membrane, further interrupting their connection with centrosome via microtubule, and 
weakening the function of LINC complex. All these result in the decrease of cell mechanical tension, and finally, the cells become more oblate and bigger. In addition, centrosome cannot dislodge from NE successfully. However, the location of centrosome determines the polarity of cell. In other words, the failure of centrosome movement may lead to the failure of cell division, or results in cell's abnormal division and further aneuploid. Cell performs protein synthesis and chromosome replication during interphase before mitosis, if failure in division, cell will be larger in size and further becomes tetraploid. In tetraploid cell, the chromosome ploidy makes its nuclear volume and N/C ratio raise, respectively, and further influence the expression level of unknown genes ${ }^{[20]}$. All of these may make cell size larger and larger. Inferior to diploid cell in stability, aneuploid and tetraploid cells are easier to die and / or in a few cases, become immortalization. Usually it is hard for aneuploid cell to grow or divide in normal tissue, however, more aneuploid cells are observed in tumor tissue, probably because gene mutation endows them more superiority to withstand the negative effect of aneuploidy. Finally, the tetraploid and aneuploidy of HeLa cells with larger size survive and be observed.

\subsection{Figures}

Cell size can be reflected indirectly via cell numbers in a fixed field under microscope, and the cell size is negative correlation to the cell number.

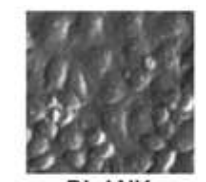

BLANK

$(10 \times 20$, unstained $)$

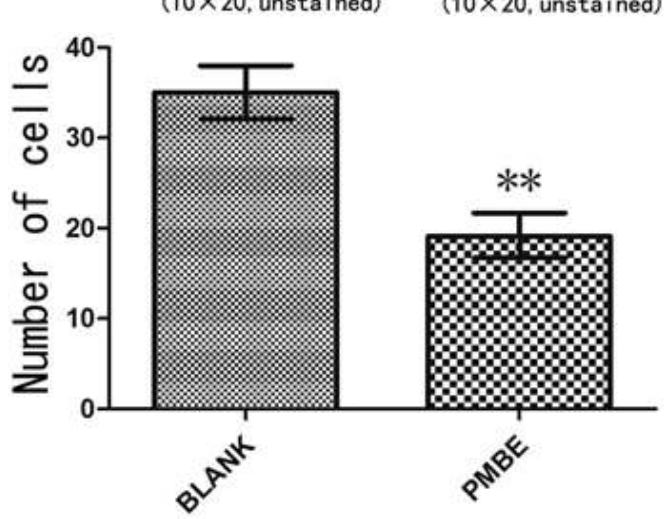

Figure 1. Effect of PMBE on the size of HeLa cells cultured in vitro $\left(\right.$ Mean $\pm \mathrm{SD} ;{ }^{\star \star} \mathrm{p}=0.002,<0.01$ versus blank group; $\mathrm{n}=3$ )

The transcription level of syne-2 is evaluated by the brightness value of bands of PCR products. The influence of PMBE on the transcription of syne-2 can be reflected via the variation of the ratio of the brightness value of syne-2 PCR band to the brightness value of GAPDH PCR band. The inset shows a representative result of the electrophoresis of respective PCR products.

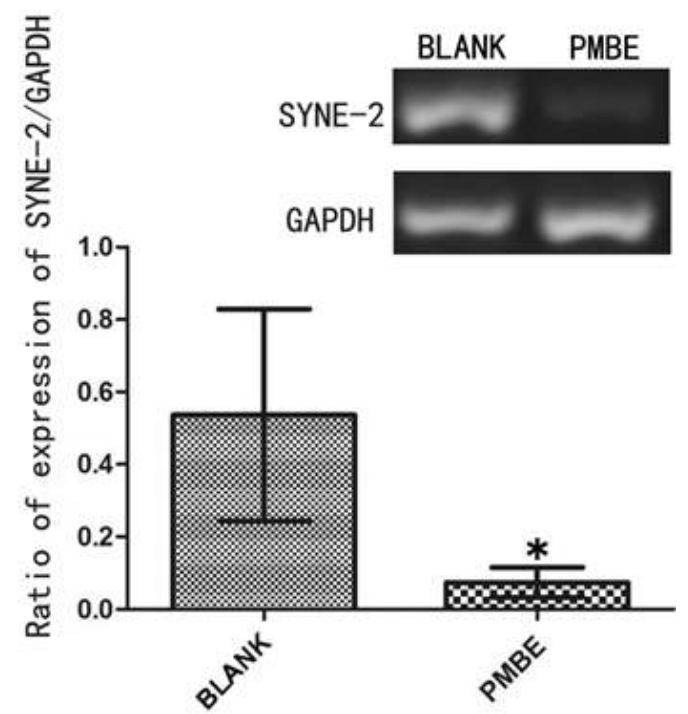

Figure 2. Impact of PMBE on the expression of syne-2 at the transcriptional level in HeLa cells (Mean $\pm \mathrm{SD} ;{ }^{\star} \mathrm{p}=0.02,<0.05$ versus blank group; $\mathrm{n}=5$ )

PMBE inhibits the transcription of syne-2 and leads to the reduction of Nesprin-2, whose location is across nuclear membranes. The polarization of centrosome is inhibited, and mitosis cannot continue. The giant tetraploid tumor cells grow and further divide into aneuploidy and/or diploid, which may help them acquire potential drug resistance.

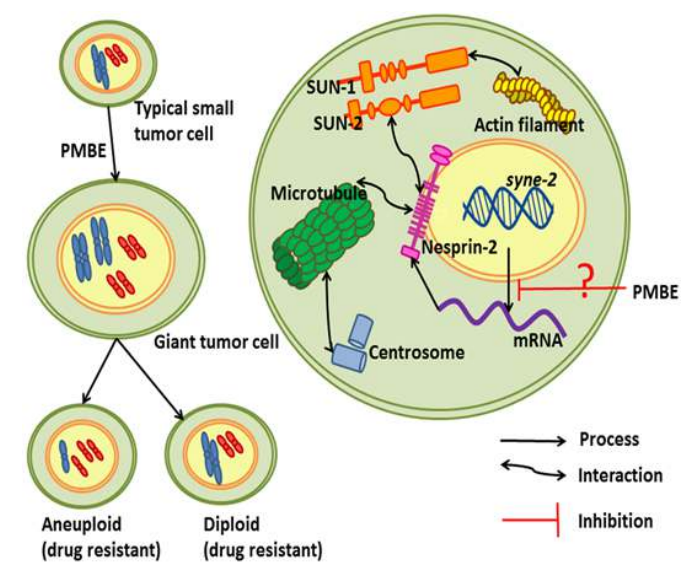

Figure 3. Hypothesis of PMBE affecting the size of HeLa cells via inhibiting syne-2 transcription

\section{Discussion}

There are more aneuploidy cells in tumor tissue. Research has shown that appearance of tetraploid cells is related to the initialization of tumor, for tetraploid is in- 
stable and tends to become aneuploidy, which may give cell immortalization ${ }^{[33]}$. This hints that it is possible for PMBE to induce chromosome abberation of HeLa cells by blocking its division via abnormal expression of Nesprin2. Nesprin-2 is an important composition of LINC, and plays a role in gene expression and cell movement. In syne-2 knock-down cells, perinuclear F-actin filaments were largely absent. In syne-2 depletion cells, keratin bundles adjacent to NE were reduced significantly or absent. The loss of Nesprin-2 or kinesin light chain-1 (KLC1), or the uncoupling of Nesprin-2 with SUN domain all will hinder centrosome move away from nuclear. Lack of Nesprin-2 will also affect the expression of KLC1 and then affects cytoskeleton and Golgi apparatus. Mice with syne-2 knockout have an increased epidermal thickness, and harbor cell polarity defects ${ }^{[19]}$. In syne-1 \& 2 depletion endothelial cells, the cellular protrusions are longer, nuclear area increase, cell migration and angiogenic loop formation decrease $\mathrm{e}^{[17]}$. All of these prove that Nesprins are crucial to nuclear shape and behavior, as well as cell polarity.

Nesprins are nuclear envelope protein. And nuclear, as the center of cell, its change will certainly influence the whole cell. As the important member of LINC complex, change of Nesprins can also affect cell shape and behaviors. For tetraploid cell, when it divides, redundant centrosomes will appear, and exactly results in cell instability. So it is reasonable to hypothesize that Nesprin-2 is the key reason for HeLa cell's size increase. In studies of inducing drug resistant tumor cells using antineoplastic agents, several kinds of resistant tumor cells are observed increase in size, including ovarian cancer cells ${ }^{[28]}$, lung cancer cells ${ }^{[29,30]}$ and murine lymphoma cells ${ }^{[23]}$, or abnormal shape of nuclear, such as gastric cancer cells ${ }^{[31]}$. Some resistant tumor cells, such as breast cancer cells, cell cycle arrest is observed ${ }^{[32]}$. These results signify that the linkage between tumor cell size and antineoplastic drug resistance may exist. GTCs show inheritable drug resistant and stronger viability ${ }^{[24-26]}$, and can be the potential reason for tumor relapse ${ }^{[27]}$.

Our primary experiment results illuminated that PMBE inhibited the expression of syne-2 at transcriptional level. This is the basis of our hypothesis. However, whether PMBE influences the translation or the activity of Nesprin2 is still unknown. Nesprin-1 \& 2 have higher isogeny, both of them link the nucleus to actin filaments by interacting with SUN1 \& 2. It has been confirmed that syne-1 knock-down has no effect on other LINC protein located in NE. As the member of LINC, location of SUN-1 \& 2 and Nesprin-2 is not influenced by the lack of Lamin A/C. If syne-1 is knocked down, the SUN-1 \& 2 and Lamin A/C will locate on NE; while if Lamin A/C is knocked down, the SUN-1 \& 2 and Nesprin-2 will still locate on $\mathrm{NE}^{[15]}$. It means members of LINC can compensate each other in some way. When Nesprin-2 decreased, as Nesprin-1 has the similar function with Nesprin-2, Nesprin-1 is possible to be increased. Whether cellular morphology is affected by the decrease of Nesprin-2 alone is uncertain. In addition, as a kind of malignant tumor cell, HeLa cell has chromosome abnormality itself, whether this abnormality will be changed by PMBE needs further validation.

Furthermore, it is necessary to measure the expression and activity of Nesprin-2 at translational and posttranslational level after treatment with PMBE. To perform karyotype analysis of HeLa cells in both PMBE group and blank group, and calculate the proportion of abnormal karyotype, respectively, is a way to verify whether the abnormality change is correlated with PMBE treatment.

Previous peer researches prove that the LINC plays an important role in cell polarization and the location of centrosome, but the underlying molecular mechanisms stand still unclear. If the hypothesis is verified, the influence of cytoskeleton members, e.g. Nesprin-2, on cell polarization and cell size will become more clarity. In our experiments, we used $154 \mu \mathrm{g} / \mathrm{mL}$ PMBE ( $\mathrm{IC}_{50}$ ) to treat HeLa cells, $50 \%$ cells were inhibited, and the other escaped from drug were highly possible to get drug resistance. Drug resistance is a vital reason for the failure in chemotherapy of malignant tumor in $\mathrm{clinic}^{[34]}$, and also a problem urgent to resolve. For over a century, clinical cancer treatment research has focused on drug development. Of note, recently, the theory of evolution by Charles Darwin has been suggested to help direct cancer treatment ${ }^{[35]}$, or so-called Darwinian Cancer Drug Program. Taking the tumor microenvironment alteration into consideration, the program suggests the existing drugs should be used more effectively than the standard continuous administration at maximum tolerated dose until the remaining cancer cells progress to a certain extent ${ }^{[36]}$. And this treatment strategy can not only maintain the control of tumor for a longer time, but also reduce the toxicity due to the significant reduction of drug dosage, patients will reduce the harm caused by toxic side effects ${ }^{[36]}$. This novel program and strategy seems to support the correctness of our preliminary findings and the potential application value of further research. Furthermore, it still needs verifying whether drug resistance is related to cell size, centrosome and chromosome karyotype. If true, it may offer novel targets for the R\&D of antineoplastic drugs.

\section{Conclusion}

In summary, we provided the primary evidence that PMBE treatment increases the size of HeLa cells and 
inhibits the expression of syne-2 in them. Based on these results, we put forward a hypothesis that PMBE regulates the size of HeLa cells via Nesprin-2 pathway. Targeting Nesprin-2 might serve as a novel promising regimen to control tumor size and antagonize the formation of drugresistance in tumors.

\section{Materials and methods}

\subsection{Cells and reagents}

HeLa cells were obtained from American Type Culture Collection. PMBE powder (Lot.1116) was purchased from N.B.C Biological Material Co.Ltd (Huzhou, Zhejiang, China). HeLa cells were cultured in Dulbecco's modified Eagle's medium (HyClone, GE Healthcare, Logan, UT, USA) added $10 \%$ heat-inactivated fetal bovine serum (HyClone, GE Healthcare, Logan, UT, USA), 100 units $/ \mathrm{mL}$ penicillin and $100 \mu \mathrm{g} / \mathrm{mL}$ streptomycin (Beyotime Biotechnology, Shanghai, China), and maintained at $37^{\circ} \mathrm{C}$ in a humidified incubator containing $5 \% \mathrm{CO}_{2}$.

\subsection{MTT assay and determinate the $50 \%$ in- hibition concentration $\left(\mathrm{IC}_{50}\right)$ of PMBE}

HeLa cells were seeded into 96-well plate and then treated with different concentrations of PMBE (40-200 $\mu \mathrm{g} / \mathrm{mL}$ ) for 48 hours. The MTT Cell Proliferation and Cytotoxicity Assay Kit was purchased from Beyotime Biotechnology (Shanghai, China). MTT assay and $\mathrm{IC}_{50}$ software were used to obtain the $\mathrm{IC}_{50}{ }^{[13]}$.

\subsection{Cell size evaluation}

HeLa cells were divided into control group (blank) and PMBE group (treated with PMBE of $\mathrm{IC}_{50}=154$ $\mu \mathrm{g} / \mathrm{mL}$ for $48 \mathrm{~h}$ ). The cell size was evaluated indirectly by counting the total cell number in a fixed area in three different fields. Photos were taken under microscope at $10 \times 20$ magnification.

\subsection{RT-PCR}

Extract total RNA from cells using TRIzol reagent (Invitrogen, Thermo Fisher Scientific, Waltham, MA, USA) and transcribe them into complementary DNA (cDNA) using an PrimeScript RT Reagent kit (TaKaRa Biotechnology, Dalian, China). PCR was used to measure the transcription level of syne-2 and GAPDH (Internal control) using PCR Amplification Kit (TaKaRa Biotechnology, Dalian, China). The PCR results were analyzed by Image $\mathrm{J} 2 \mathrm{x}$ software and Adobe Photoshop. Measure the brightness value of band of syne-2 and GAPDH of each group, respectively, and then calculate the ratio of syne-2/GAPDH.

\subsection{Statistical analysis}

Results are presented as mean \pm S.D. of $n$ independent experiments. Statistical significance was assessed using analysis of variance. Subsequent pair-wise comparisons of specific means were assessed using the Student's t test. $\mathrm{p}<0.05$ was considered statistically significant.

\section{Author Contributions}

Conceptualization, Y.Y. Cui; methodology, X.L. Zhang, Y.Y. Li and J. Feng; validation, X.L. Zhang, M.Q. Li, Y.Y. Li and J. Feng; formal analysis, X.L. Zhang. and M.Q. Li; data curation, Y.Y. Cui; writing-original draft preparation, X.L. Zhang; writing-review and editing, M.Q. Li, Y.Y. Li and J. Feng.; supervision, Y.Y. Cui; project administration, Y.Y. Cui; funding acquisition, Y.Y. Cui.

\section{Funding}

This project was funded by National University Students Innovation Training Program, grant number: 1500107070; Yangfan Project of Tongji University School of Medicine, grant number: 2012YF05; Fundamental Research Funds for the Central Universities, grant number: 22120180190 and Shanghai Educational Research Project grant number: C19154.

\section{Acknowledgements}

Authors would like to convey their thanks to the staff of the Key Laboratory of Arrhythmias, Ministry of Education (Tongji University) for their technical assistance, and the reviewers for their helpful suggestions, which improve the quality of the paper considerably.

\section{Conflicts of interest}

There was no conflict of interest.

\section{References}

[1] Cui YY, Xie H and Wang JF. Potential biomedical properties of Pinus massoniana bark extract. Phytotherapy Research, 2005, 19(1): 34-38. https://doi.org/10.1002/ptr.1619

[2] Cui YY, Xie H, Qi KB, et al. Effects of Pinus massoniana bark extract on cell proliferation and apoptosis of human hepatoma BEL-7402 cells. World Journal of Gastroenterology, 2005, 11(34): 5277-5282. https://doi.org/10.3748/wjg.v11.i34.5277

[3] Yang ZQ, Chen H, Tan JH, et al. Cloning of three genes involved in the flavonoid metabolic pathway and their expression during insect resistance in Pinus massoniana Lamb. Ge- 
netics \& Molecular Research, 2016, 15(4): gmr15049332. https://pubmed.ncbi.nlm.nih.gov/28081282

[4] Zeng YX, Wang S, Wei L, et al. Proanthocyanidins: Components, Pharmacokinetics and Biomedical Properties. American Journal of Chinese Medicine, 2020, 48(4): 813-869. https://doi.org/10.1142/S0192415X2050041X

[5] Wu CL, Feng DR, Ma HL, et al. Effect of Pinus massoniana bark extract on IFN- $\gamma$-induced ICAM-1 expression in HaCaT human keratinocytes. Journal of Ethnopharmacology, 2009, 122 (1): 48-53. https://doi.org/10.1016/j.jep.2008.11.024

[6] Feng J, Zhang XL, Li YY, et al. Pinus massoniana Bark Extract: Structure-Activity Relationship and Biomedical Potentials. The American Journal of Chinese Medicine, 2016, 44(8): 1559-1577. https://doi.org/10.1142/S0192415X16500877

[7] Xu S, Zhang S, Wang X, et al. Effect of Pinus massoniana Lamb. bark extract on lytic cycle of Epstein-Barr virus. Indian Journal of Experimental Biology, 2012, 50(10): 708713.

https://doi.org/10.7440/colombiaint83.2015.04

[8] Mao P, Zhang E, Chen Y, et al. Pinus massoniana bark extract inhibits migration of the lung cancer A549 cell line. Oncology Letters, 2017, 13(2): 1019-1023. https://doi.org/10.3892/ol.2016.5509

[9] Ma H, Liu B, Feng D, et al. Pinus massoniana bark extract selectively induces apoptosis in human hepatoma cells, possibly through caspase-dependent pathways. International Journal of Molecular Medicine, 2010, 25(5): 751-759. https://pubmed.ncbi.nlm.nih.gov/20372819

[10] Li YY, Feng J, Zhang XL, et al. Pine bark extracts: nutraceutical, pharmacological, and toxicological evaluation.Journal of Pharmacology \& Experimental Therapeutics, 2015, 353(1): 9-16.

https://doi.org/10.1124/jpet.114.220277

[11] Lin BW, Gong CC, Song HF, et al. Effects of anthocyanins on the prevention and treatment of cancer. British Journal of Pharmacology, 2017, 174(11): 1226-1243. https://doi.org/10.1111/bph.13627

[12] Wu DC, Li S, Yang DQ, et al. Effects of Pinus massoniana bark extract on the adhesion and migration capabilities of HeLa cells. Fitoterapia, 2011, 82(8): 1202-1205. https://doi.org/10.1016/j.fitote.2011.08.008

[13] Li YY, Feng J, Zhang XL, et al. Effects of Pinus massoniana bark extract on the invasion capability of HeLa cells. Journal of Functional Foods, 2016, 24: 520-526. https://doi.org/10.1016/j.jff.2016.04.030

[14] Zhao CX, Cui YY and Chen YH. Progress of The Research on Nesprins. Progress in Biochemistry and Biophysics, 2014, 41(4): 337-344.

http://www.pibb.ac.cn/pibbcn/ch/reader/create_pdf.aspx?fil e_no $=20130186 \&$ flag $=1 \&$ journal $\_i d=$ pibben

[15] Zhou C, Rao L, Shanahan CM, et al. Nesprin-1/2: roles in nuclear envelope organisation, myogenesis and muscle disease. Biochemical Society Transactions, 2018, 46(2): 311320.

https://doi.org/10.1042/BST20170149
[16] Elvira I, Alessia C, Robin F, et al. LINC complex-Lis1 interplay controls MT1-MMP matrix digest-on-demand response for confined tumor cell migration. Nature Communications, 2018, 9(1): 1-13. https://doi.org/10.1038/s41467-018-04865-7

[17] King SJ, Nowak K, Suryavanshi N, et al. Nesprin-1 and nesprin-2 regulate endothelial cell shape and migration. Cytoskeleton, 2014, 71(7): 423-434. https://doi.org/10.1002/cm.21182

[18] Matsumoto A, Hieda M, Yokoyama Y, et al. Global loss of a nuclear lamina component, lamin $\mathrm{A} / \mathrm{C}$, and LINC complex components SUN1, SUN2, and nesprin-2 in breast cancer. Cancer Medicine, 2015, 4(10): 1547-1557. https://doi.org/10.1002/cam4.495

[19] Schneider M, Lu W, Neumann S, et al. Molecular mechanisms of centrosome and cytoskeleton anchorage at the nuclear envelope. Cellular \& Molecular Life Sciences Cmls, 2011, 68(9): 1593-1610. https://doi.org/10.1007/s00018-010-0535-z

[20] Björklund M. Cell size homeostasis: Metabolic control of growth and cell division. Biochimica Et Biophysica Acta Molecular Cell Research, 2019, 1866(3): 409-417. https://doi.org/10.1016/j.bbamcr.2018.10.002

[21] Ginzberg MB, Kafri R and Kirschner M. On being the right (cell) size. Science, 2015, 348(6236): 771. https://doi.org/10.1126/science.1245075

[22] Dungrawala H, Hua H, Wright J, et al. Identification of new cell size control genes in S. cerevisiae. Cell Division, 2012, 7(24): 1-13.

https://doi.org/10.1186/1747-1028-7-24

[23] Horbay R, Boiko N, Shlyakhtina Y, et al. Giant cancer cells: overcoming drug resistance again and again. Total overview, 2012.

https://doi.org/10.13140/2.1.3864.4804

[24] Montgomery C, Couch C, Emory CL, et al. Giant Cell Tumor of Bone: Review of Current Literature, Evaluation, and Treatment Options. Journal of Knee Surgery, 2019, 32(4): 331-336. https://doi.org/10.1055/s-0038-1675815

[25] Amend SR, Torga G, Lin KC, et al. Polyploid giant cancer cells: Unrecognized actuators of tumorigenesis, metastasis, and resistance. Prostate, 2019, 79(13): 1489-1497. https://doi.org/10.1002/pros.23877

[26] Vitale I, Jemaà M, Senovilla L, et al. Involvement of p38alpha in the mitotic progression of p53(-/-) tetraploid cells. Cell Cycle, 2010, 9(14): 2823-2829. https://doi.org/10.4161/cc.9.14.12254

[27] Yeung YT, Yin S, Lu B, et al. Losmapimod Overcomes Gefitinib Resistance in Non-small Cell Lung Cancer by Preventing Tetraploidization. EBioMedicine, 2018, 28: 51-61. https://doi.org/10.1016/j.ebiom.2018.01.017

[28] Fan P, Li HX, Wu YM, et al. Biological characteristics of paclitaxel-resistance ovarian cancer cell lines and cryopreservation methods. Cancer Research on Prevention and Treatment, 2015, 42(4): 334-339. https://doi.org/10.3971/j.issn.1000-8578.2015.04.004

[29] Liu X, Jia YH, Zhang K, et al. Establishment of crizotinibresistant non-small cell lung cancer cell line NCI-H3122 
and preliminary study of resistance related miRNAs. Journal of Third Military Medical University, 2016, 38(7): 687692.

http://aammt.tmmu.edu.cn/Upload/rhtml/201511024.htm

[30] Yvorel V, Patoir A, Casteillo F, et al. PD-L1 expression in pleomorphic, spindle cell and giant cell carcinoma of the lung is related to TTF-1, p40 expression and might indicate a worse prognosis. PLoS One, 2017, 12(7): e0180346. https://doi.org/10.1371/journal.pone.0180346

[31] Du JP, Chen P, Fei HX, et al. Establishment of adriamycinresistant and cisplatin-resistant gastric cancer cell lines and assessment on their sustainability of drug resistance. Journal of Gastroenterology and Hepatology, 2007, 16(4): 368-372. https://doi.org/10.4028/0-87849-470-7.369

[32] Gul A, Leylandjones B, Dey N, et al. A combination of the PI3K pathway inhibitor plus cell cycle pathway inhibitor to combat endocrine resistance in hormone receptor-positive breast cancer: a genomic algorithm-based treatment approach. American Journal of Cancer Research, 2018, 8(12): 2359-2376.

http://www.ajcr.us/files/ajcr0088398.pdf
[33] Wang Y, Yuan BB and Yue TF. Research progress of polyploidy and cancer. Journal of International Obstetrics and Gynecology, 2010, 37(6): 420-426. (In Chinese)

[34] Cui YY. Cancer, mankind's challenge. Current Cancer Reports, 2019, 1(1): 1-5. https://doi.org/10.25082/CCR.2019.01.001

[35] Robert G and Christopher W. Cancer treatment innovators discover Charles Darwin. Evolution, Medicine, and Public Health, 2019, 2019(1): 108-110. https://doi.org/10.1093/emph/eoz018

[36] DeGregori J and Gatenby R. Darwin's ideas on evolution drive a radical new approach to cancer drug use. Principle of evolution and natural selection drive a radical new approach to drugs and prevention strategies. Scientific American, 2019, 321(2): 52. https://doi.org/10.1038/scientificamerican0819-52

(Edited by Snowy Wang) 